\title{
Endoscopic treatments prior to urethroplasty: trends in management of urethral stricture disease
}

\author{
Matthew J. Moynihan ${ }^{1 *}$, Bryan Voelzke ${ }^{2}$, Jeremy Myers ${ }^{3}$, Benjamin N. Breyer ${ }^{4}$, Bradley Erickson ${ }^{5}$, Sean P. Elliott ${ }^{6}$,
} Nejd Alsikafi', Jill Buckley ${ }^{8}$, Lee Zhao ${ }^{9}$, Thomas Smith $^{10}$ and Alex J. Vanni ${ }^{1}$

\begin{abstract}
Background: To determine if the number of endoscopic treatments of urethral stricture disease (USD) prior to urethroplasty has changed in the context of new AUA guidelines on management of USD. In addition to an increase in practicing reconstructive urologists and published reconstructive literature, the AUA guidelines regarding the management of male USD were presented in May 2016, advocating consideration of urethroplasty in patients with 1 prior failed endoscopic treatment.

Methods: A retrospective review of a prospectively maintained, multi-institutional urethral stricture database of high volume, geographically diverse institutions was performed from 2006 to 2017. We performed a review of relevant literature and evaluated pre-urethroplasty endoscopic treatment patterns prior to and after the AUA male stricture guideline.

Results: 2964 urethroplasties were reviewed in 10 institutions. There was both a decrease in the number of endoscopic treatments prior to urethroplasty in the pre-May 2016 compared to post-May 2016 cohorts both for overall urethroplasties (2.3 vs 1.6, $P=0.0012$ ) and a gradual decrease in the number of pre-urethroplasty endoscopic treatments over the entire study period.

Conclusion: There was a decrease in the number of endoscopic treatments of USD prior to urethroplasty in the observed period of interest. Declining endoscopic USD management is not likely to be a reflection of a solely unique influence of the guidelines as endoscopic treatment decreased over the entire study period. Further research is needed to determine if there will be a continued trend in the declining use of endoscopic treatment and elucidate the barriers to earlier urethroplasty in patients with USD.
\end{abstract}

Keywords: Urethroplasty, Urethral stricture guidelines, Endoscopic treatment, Urethral stricture

\section{Background}

Urologists play a critical role in the evaluation and management of urethral stricture disease (USD), which accounts for nearly 1.5 million office visits per year [1]. This condition has most commonly been managed with

\footnotetext{
* Correspondence: matthew.moynihan@lahey.org

'Department of Urology, Lahey Hospital and Medical Center, 41 Mall Rd, Burlington, MA 01805, USA

Full list of author information is available at the end of the article
}

endoscopic treatments such as urethral dilation and direct vision internal urethrotomy (DVIU) [2]. However, endoscopic management has poor success with stricture recurrence rates between 50 and 92\% [3, 4]. Additionally, multiple failed endoscopic treatments can make subsequent urethral reconstruction more challenging [5-7]. As such, a shift in management towards earlier urethroplasty has been seen in recent years, which has

(c) The Author(s). 2020 Open Access This article is licensed under a Creative Commons Attribution 4.0 International License, which permits use, sharing, adaptation, distribution and reproduction in any medium or format, as long as you give appropriate credit to the original author(s) and the source, provide a link to the Creative Commons licence, and indicate if changes were made. The images or other third party material in this article are included in the article's Creative Commons licence, unless indicated otherwise in a credit line to the material. If material is not included in the article's Creative Commons licence and your intended use is not permitted by statutory regulation or exceeds the permitted use, you will need to obtain permission directly from the copyright holder. To view a copy of this licence, visit http://creativecommons.org/licenses/by/4.0/ The Creative Commons Public Domain Dedication waiver (http://creativecommons.org/publicdomain/zero/1.0/) applies to the data made available in this article, unless otherwise stated in a credit line to the data. 
higher success rates and better reported patient outcomes [8].

Unfortunately, consensus on the management of male USD has historically been hindered by a lack of definitive practice recommendations. In fact, despite the available evidence, a retrospective single institutional review by Granieri and Peterson saw no measurable change in practice patterns before referral to urethroplasty from 1996 to 2010 [9]. In order to more definitively set recommendations for the urologic community, the American Urological Association (AUA) published its first set of evidence based guidelines regarding male urethral stricture disease in 2016 [10]. The guidelines are notable for their recommendations for earlier urethroplasty and avoidance of repeated endoscopic management for recurrent urethral strictures. Specifically, the AUA male urethral stricture guideline recommends that patients should be offered urethroplasty after failed endoscopic management (Guideline 11) and also for fossa navicularis, penile, bulbar strictures $>2 \mathrm{~cm}$, and recurrent bulbar strictures (Guidelines 13-16) [10]. However, it is unclear if the new guideline has decreased the number of endoscopic treatments prior to referral for urethroplasty. Our objective is to determine if there has been a decrease in the use of endoscopic treatment of USD in high volume reconstructive practices over time and whether the AUA guideline has accelerated this impact. We hypothesize that the number of endoscopic treatments prior to urethroplasty in the cohort has decreased over time and that the guideline has further decreased the use of endoscopic treatment prior to referral.

\section{Methods}

Institutional review board approval was obtained for this study (Lahey Hospital and Medical Center Institutional Review Board \#20193211). We performed a literature review of publications describing factors driving changes in the utilization of urethroplasty. We then conducted a retrospective review of a prospectively maintained multiinstitutional database of all patients who underwent a urethroplasty performed by a total of 10 surgeons between years 2006-2017. Patient demographics, length of stricture, etiology, and pre-operative endoscopic interventions were recorded. Either DVIU or urethral dilation were considered to be endoscopic treatment. To determine if pre-urethroplasty endoscopic treatment patterns prior to referral changed after the May 2016 AUA stricture guidelines, the number of endoscopic treatments prior to urethroplasty were recorded and grouped into pre-May 2016 and post-May 2016 cohorts. Statistics were performed with Chi-square tests and t-tests where appropriate.

\section{Results}

A total of 2964 urethroplasties performed by 10 surgeons were reviewed that had sufficient data for analysis. Patient demographics can be found in Table 1. Those undergoing posterior urethroplasty were statistically significantly more likely to be older, hypertensive, hyperlipidemic, have a history of malignancy, and history of pelvic radiation (all $P<0.0001$ ).

There was no difference in overall mean stricture length between cohorts $(3.8 \mathrm{~cm}$ vs $3.7 \mathrm{~cm}, P=0.5928)$. Looking specifically at etiology of anterior urethroplasties, idiopathic strictures were the most common etiology (43\%). The pre-May 2016 cohort had a greater proportion of traumatic $(15.2 \%$ vs $10.5 \%, P=0.0022)$ strictures. The post-May 2016 cohort had a greater proportion of anterior urethroplasties performed for iatrogenic $(22.0 \%$ vs $16.2 \%, P=0.0006)$ and failed hypospadias $(9.76 \%$ vs $6.3 \%, P=0.0027)$ etiologies. There was no significant difference in proportion of idiopathic, infectious, lichen sclerosus, or radiation as the etiology of the anterior USD between the two cohorts (Table 2).

Overall average number of endoscopic treatments prior to urethroplasty for the entire cohort was 2.15 $(\mathrm{SD}=4.887)$. Endoscopic treatment prior to urethroplasty is less common in patients undergoing posterior urethral reconstruction compared to anterior ( 0.38 vs 2.0, $P<0.0001)$. With regard to anterior urethroplasties, a majority of patients $(69.8 \%)$ had at least one endoscopic treatment prior to urethroplasty. For all urethroplasties, $37.7 \%$ had at least 2 endoscopic treatments (range 0-91) prior to undergoing urethroplasty.

There was a significant decrease in the average endoscopic pre-urethroplasty treatments prior to referral between the pre-May 2016 and post-May 2016 cohorts for both overall urethroplasties $(2.3$ vs $1.6, P=0.0012)$ and specifically anterior urethroplasties (2.6 vs $1.9, P=$ $0.0026)$. Notably however, there has been a gradual decrease in the number of pre-urethroplasty endoscopic treatments over the study period (Fig. 1). When analyzed individually, only one surgeon had an increase in average overall pre-urethroplasty endoscopic treatments of 0.09 , although not statistically significant $(P=0.9157)$ (Fig. 1$)$.

A subgroup analysis of anterior urethral strictures $\leq 2$ $\mathrm{cm}$ was performed. This cohort was analyzed because the AUA USD guidelines give the option of initial endoscopic management for meatal, fossa navicularis, and bulbar urethral strictures $\leq 2 \mathrm{~cm}$, and thereby would act as a surrogate. There were 677 patients in the pre-May 2016 subset and 218 in the post-May 2016 subset with sufficient data for analysis. There was no statistically significant difference between the pre- and post-May 2016 groups with regard to median (1 vs 1 ) or mean endoscopic treatments (2.04 vs $1.6, P=0.1225)$, respectively. 
Table 1 Patient demographics and characteristics

\begin{tabular}{|c|c|c|c|}
\hline & Anterior Urethroplasty & Posterior Urethroplasty & Total \\
\hline Participants, $n$ & 2,581 & 383 & 2,964 \\
\hline \multicolumn{4}{|l|}{ Patient Demographics } \\
\hline Mean age (range), years & $47.2(0-91)$ & $56.7(8-86)$ & $48.4(0-91)$ \\
\hline Mean BMI (range) & $30.1(11-64)$ & $27.9(10-52)$ & $29.8(10-64)$ \\
\hline \multicolumn{4}{|l|}{ Co-morbid Conditions } \\
\hline Diabetes, $n$ (\%) & $345(13.4 \%)$ & $58(15.1 \%)$ & $403(13.6 \%)$ \\
\hline Hypertension, $n(\%)$ & $813(31.5 \%)$ & $177(46.2 \%)$ & $990(33.4 \%)$ \\
\hline Hyperlipidemia, $n(\%)$ & $516(20.0 \%)$ & $121(31.6 \%)$ & $637(21.5 \%)$ \\
\hline Coronary Artery Disease, $n(\%)$ & $70(2.7 \%)$ & $16(4.2 \%)$ & $86(2.9 \%)$ \\
\hline COPD, $n(\%)$ & $57(2.2 \%)$ & $12(3.1 \%)$ & $69(2.3 \%)$ \\
\hline Peripheral Vasc Disease, $n(\%)$ & $36(1.4 \%)$ & $6(1.6 \%)$ & $42(1.4 \%)$ \\
\hline History of Malignancy, $n$ (\%) & $187(7.2 \%)$ & $135(35.2 \%)$ & $322(10.9 \%)$ \\
\hline Prior Pelvic Radiation, $n$ (\%) & $20(0.8 \%)$ & $140(36.6 \%)$ & $160(5.4 \%)$ \\
\hline
\end{tabular}

\section{Discussion}

There was a decrease in the number of endoscopic treatments of USD prior to urethroplasty in the observed period of interest. Declining endoscopic USD management is not likely to be a reflection of a solely unique influence of the guidelines as endoscopic treatment decreased over the entire study period. Interpretation of this finding however must be guarded as it seems to be a downward trend consistent with an overall change in practice patterns over the observed time period. Admittedly, previous reports have demonstrated a downward trend in the use of endoscopic management accompanied by an increase in urethroplasties $[11,12]$, and therefore the lack of a significant inflection point at time of guideline release in our cohort suggests the guideline is not a unique influence.
The results from this study still suggest that urethral dilation and DVIU remain the most common treatment modalities for USD management, even in areas where patients have local access to fellowship trained reconstructive surgeons. The observed number of patients with at least one endoscopic procedure in our study (69.8\%) was consistent with the $65.5 \%$ rate found in a large review of Veterans Affairs data from 1999 to 2013 [13]. However, inconsistent with prior studies was our observation that significantly fewer men reported $\geq 2$ endoscopic procedures prior to urethroplasty (37.7\%) than previously reported series, where close to $70 \%$ of patients fell into this category $[9,14]$. While this change may also be related to publication of the guidelines, alternative explanations include the expansion of fellowship trained reconstructive surgeons who can now

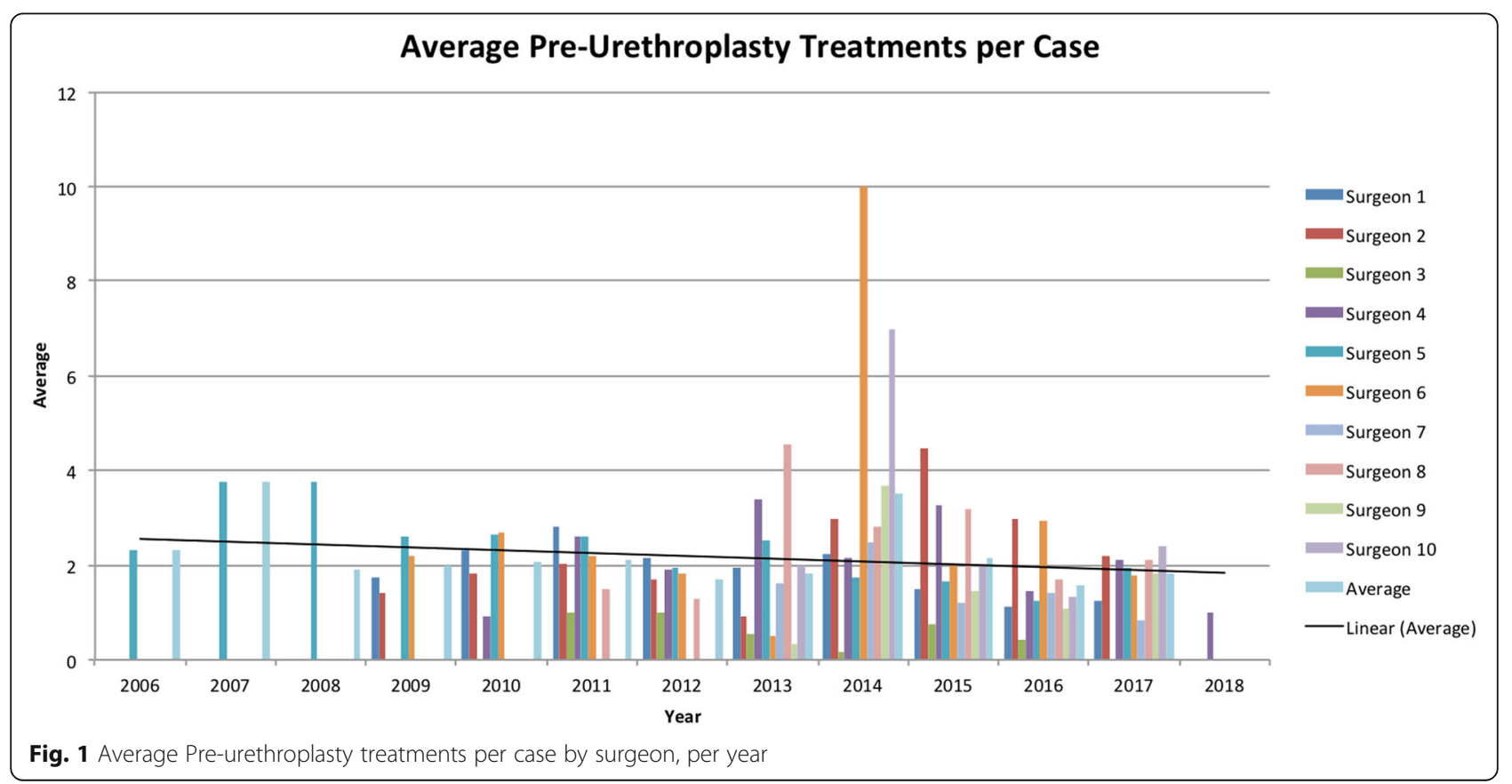


Table 2 Etiology of anterior urethral stricture disease by cohort with respect to release of AUA urethral stricture disease guidelines

\begin{tabular}{|c|c|c|c|c|c|}
\hline \multicolumn{6}{|c|}{ ANTERIOR STRICTURE ETIOLOGY } \\
\hline & & Cases (n) & $\%$ of Total & $95 \% \mathrm{Cl}$ & P-value \\
\hline \multirow[t]{3}{*}{ Idiopathic } & & & & $-0.9686-7.6084$ & 0.1347 \\
\hline & Pre-5/2016 & 863 & $43.83 \%$ & & \\
\hline & Post-5/2016 & 274 & $40.53 \%$ & & \\
\hline \multirow[t]{3}{*}{ latrogenic } & & & & $2.4284-9.4708$ & 0.0006 \\
\hline & Pre-5/2016 & 319 & $16.20 \%$ & & \\
\hline & Post-5/2016 & 149 & $22.04 \%$ & & \\
\hline \multirow[t]{3}{*}{ Trauma } & & & & $1.7815-7.4075$ & 0.0022 \\
\hline & Pre-5/2016 & 300 & $15.24 \%$ & & \\
\hline & Post-5/2016 & 71 & $10.50 \%$ & & \\
\hline \multicolumn{4}{|c|}{ Failed Hypospadias } & $1.1346-6.1210$ & 0.0027 \\
\hline & Pre-5/2016 & 124 & $6.30 \%$ & & \\
\hline & Post-5/2016 & 66 & $9.76 \%$ & & \\
\hline \multirow[t]{3}{*}{ Infectious } & & & & $-0.2940-2.5505$ & 0.1474 \\
\hline & Pre-5/2016 & 37 & $1.88 \%$ & & \\
\hline & Post-5/2016 & 19 & $2.81 \%$ & & \\
\hline \multicolumn{4}{|c|}{ Lichen Sclerosis } & $-2.2312-2.2173$ & 0.8875 \\
\hline & Pre-5/2016 & 137 & $6.96 \%$ & & \\
\hline & Post-5/2016 & 46 & $6.80 \%$ & & \\
\hline \multirow[t]{3}{*}{ Radiation } & & & & $-0.6775-1.7660$ & 0.5389 \\
\hline & Pre-5/2016 & 31 & $1.57 \%$ & & \\
\hline & Post-5/2016 & 13 & $1.92 \%$ & & \\
\hline
\end{tabular}

support high volume urethroplasty practices, literature published prior to the release of the AUA guidelines demonstrating the cost-effective benefit of primary reconstruction over endoscopic management $[15,16]$, and studies emphasizing the futility of repeated endoscopic treatments [17].

Our pre-May 2016 cohort finding of an average of 2.3 pre-urethroplasty treatments for all urethroplasties and 2.6 pre-urethroplasty treatments for anterior strictures is consistent with previous studies performed during the time period [18]. Altogether, we did find a decrease in average number of pre-urethroplasty endoscopic treatments after publication of the AUA guidelines both for overall urethroplasties and anterior urethroplasties. As mentioned, in addition to the AUA guidelines, the decrease in multiple endoscopic treatments may be due to the presence of a fellowship trained reconstructive surgeon available for tertiary referral for urologists who would otherwise treat USD by endoscopic means. More reconstructive urologists are graduating from fellowships, and both younger urologists and those academically affiliated are more likely to manage strictures with urethroplasty than endoscopic treatments [19].
Admittedly, the reconstructive surgeons in our study group have been present in their geographic region for years and thus the decrease in endoscopic treatments seen after May 2016 would not be due to a new expert in the region, but rather could be explained by community urologists referring patients for urethroplasty earlier than in the past in response to the AUA guideline. We additionally note that our conclusions are limited secondary to the shorter follow up of patients after the 2016 AUA guidelines for the time period investigated in our study.

We considered urethral stricture length and stricture etiology as potential confounders of our study. However, there was no significant difference in average stricture length or in patients with lichen sclerosus for all urethroplasties between the two cohorts under investigation, which further strengthens our findings. Our results further demonstrate that the two cohort groups are fairly similar with regard to stricture etiology and therefore etiology is not likely the underlying reason for a difference in endoscopic treatments.

There are several strengths of this study that make it uniquely informative. For example, this is the largest 
study to look specifically at this trend with a granularity regarding patient, operative, and stricture characteristics not available in many national database sample studies since the publication of the AUA guidelines, which thereby serves to make the results more informative. A Nationwide Inpatient Sample by Buckley et al. in 2016 corroborated a similar trend to what we have shown, however our prospective database gives particular insights into these findings that can serve to tailor future research and guide more accurate conclusions [20]. The granularity of our study demonstrates that even in areas served with fellowship trained reconstructive urologists, endoscopic management of USD continues to be the dominant treatment, and thus continued research is needed to determine the barriers to earlier urethroplasty. Though, even with the new AUA guideline and trends among graduating urologists, we would be remiss not to note the significant geographic disparity in urologic reconstructive expertise that influences USD management that our study does not accurately capture [21].

Endoscopic treatments such as dilation and DVIU remain a mainstay of initial treatment for short urethral strictures. Our study demonstrates a change in practice patterns over the observed time period that progressively favor urethroplasty as an intervention with a higher long-term success rate that should be integrated earlier into USD management. The lack of a clear inflection point at the time of AUA USD guidelines release date does not necessarily infer a lack of impact, but rather suggests that guidelines continue to provide further evidence of the success of earlier referral for urethroplasty that we have shown is serving to propagate a change in contemporary practice patterns and contribute to increased utilization of urethroplasty.

\section{Conclusion}

The number of endoscopic treatments of USD prior to referral for urethroplasty has decreased over the last decade and continues since development of the AUA male urethral stricture guideline. Despite this decline, there was no significant inflection point in the overall trend at the time of AUA publication. Further research is needed to determine further trends in the declining use of endoscopic treatment, examine long-term effects on patient outcomes with earlier referral for urethroplasty, and elucidate the barriers to earlier urethroplasty in patients with USD.

\footnotetext{
Abbreviations

AUA: American Urological Association; USD: urethral stricture disease; DVIU: direct vision internal urethrotomy
}

\section{Authors' contributions}

MM was involved in protocol/project development, data management, data analysis, manuscript writing/editing. AV was similarly involved in protocol/ project development, data management, data analysis, and manuscript writing/editing. BV, JM, BB, BE, SE, NA, JB, LZ, and TS were all involved in data collection and manuscript writing/editing. All authors read and approved the final manuscript.

\section{Funding}

No funding was provided for the conduct of this study.

\begin{abstract}
Availability of data and materials
The data that support the findings of this study are available from the Trauma and Urologic Reconstructive Network of Surgeons (TURNS) but restrictions apply to the availability of these data, which were used under license for the current study, and so are not publicly available. Data are however available from the authors upon reasonable request and with permission of TURNS.
\end{abstract}

\section{Ethics approval and consent to participate}

All procedures performed in studies involving human participants were in accordance with the ethical standards of the institutional and/or national research committee (include name of committee + reference number) and with the 1964 Helsinki declaration and its later amendments or comparable ethical standards.

Consent for publication

Not applicable.

\section{Competing interests}

The authors declare they have no competing interests.

\section{Author details}

'Department of Urology, Lahey Hospital and Medical Center, 41 Mall Rd, Burlington, MA 01805, USA. ${ }^{2}$ Spokane Urology, Spokane, Washington, USA. ${ }^{3}$ University of Utah, Salt Lake City, UT, USA. ${ }^{4}$ University of California - San Francisco, San Francisco, California, USA. ${ }^{5}$ University of lowa, lowa City, lowa, USA. ${ }^{6}$ University of Minnesota, Minneapolis, MN, USA. 'Uropartners, Gurnee, IL, USA. ${ }^{8}$ University of California - San Diego, San Diego, California, USA. ${ }^{9}$ New York University, Langone Medical Center, New York City, New York, USA. ${ }^{10}$ Baylor College of Medicine, Houston, TX, USA.

Received: 7 December 2019 Accepted: 4 June 2020

Published online: 13 June 2020

References

1. Santucci RA, Joyce GF, Wise M. Male urethral stricture disease. J Urol. 2007; 177(5):1667-74.

2. Anger JT, Buckley JC, Santucci RA, et al. Trends in stricture management among male Medicare beneficiaries: underuse of Urethroplasty? J Urol. 2011;77:481-5.

3. Santucci $R$, Eisenberg L. Urethrotomy has much lower success rate than previously reported. J Urol. 2010;183(5):1859-62.

4. Heyns CF, Steenkamp JW, De Kock MLS, et al. Treatment of male urethral strictures: is repeated dilation or internal urethrotomy useful? J Urol. 1998; 160(2):356-8.

5. Veeratterapillay R, Pickard RS. Long-term effect of urethral dilation and internal urethrotomy for urethral strictures. Curr Op Urol. 2012;22(6):467-73.

6. Greenwell TJ, Castle C, Andrich DE, et al. Repeat Urethrotomy and dilation for the treatment of urethral stricture are neither clinically effective nor costeffective. J Urol. 2004;172(1):275-7.

7. Hudak SJ, Atkinson TH, Morey AF. Repeat transurethral manipulation of bulbar urethral strictures is associated with increased stricture complexity and prolonged disease duration. J Urol. 2012;187(5):1691-5.

8. Blaschko SD, Harris CR, Zaid UB, et al. Trends, utilization, and immediate perioperative complications of Urethroplasty in the United States: data from the National Inpatient Sample 2000-2010. J Urol. 2015;85(5):1190-4.

9. Granieri MA, Peterson AC. The management of bulbar urethral stricture disease before referral for definitive repair: have practice patterns changed? J Urol. 2014;84(4):946-9. 
10. Wessels $\mathrm{H}$, Angermeier KW, Elliot SP, et al. Male urethral stricture. AUA Clinical Guideline. Linthicum: American Urological Association Education and Research, Inc; 2016.

11. Bullock TL, Brandes SB. Adult anterior urethral strictures: a national practice patterns survey of board certified urologists in the United States. J Urol. 2007;177(2):685-90.

12. Burks FN, Salmon SA, Smith AC, et al. Urethroplasty: a geographic disparity in care. J Urol. 2012;187(6):2124-7.

13. Lacey JM, Cavallini M, Bylund JR, et al. Trends in the management of male urethral stricture disease in the veteran population. Urology. 2014;84(6): 1506-10.

14. McLaughlin MD, Thrasher JB, Celmer A, et al. Buccal mucosal urethroplasty in patients who had multiple previous procedures. Urology. 2006;68(6): $1156-9$

15. Rourke KF, Jordan GH. Primary urethral reconstruction: the cost minimized approach to the bulbous urethral stricture. J Urol. 2005;173(4):1206-10.

16. Wright JL, Wessells $H$, Nathens $A B$, et al. What is the most cost-effective treatment for 1 to 2-cm bulbar urethral strictures: societal approach using decision analysis. Urology. 2006;67(5):889-93.

17. Buckley JC, Heyns C, Gilling P, et al. SIU/ICUD consultation on urethral strictures: dilation, internal Urethrotomy, and stenting of male anterior urethral strictures. Urology. 2014;83(3):S18-22.

18. Kane CJ, Tarman GJ, Summerton DJ, et al. Multi-institutional experience with buccal mucosa onlay urethroplasty for bulbar urethral reconstruction. J Urol. 2002;167(3):1314-7.

19. Liu JS, Hofer MD, Oberlin DT, et al. Practice patterns in the treatment of urethral stricture among american urologists: a paradigm change? Urology. 2015;86(4):830-4.

20. Buckley JC, Patel N, Wang S, Liss M. National trends in the management of urethral stricture disease: a 14-year survey of the nationwide inpatient sample. Urol Pract. 2016;1:315-20.

21. Santucci RA. The reconstructive urology workforce: present and future. Transl Androl Urol. 2014:3(2):205-8.

\section{Publisher's Note}

Springer Nature remains neutral with regard to jurisdictional claims in published maps and institutional affiliations.

Ready to submit your research? Choose BMC and benefit from:

- fast, convenient online submission

- thorough peer review by experienced researchers in your field

- rapid publication on acceptance

- support for research data, including large and complex data types

- gold Open Access which fosters wider collaboration and increased citations

- maximum visibility for your research: over $100 \mathrm{M}$ website views per year

At $\mathrm{BMC}$, research is always in progress.

Learn more biomedcentral.com/submissions 